\title{
Oxylipins in cerebrospinal fluid in clinically isolated syndrome and relapsing remitting multiple sclerosis
}

Irene Håkansson, Sandra Gouveia-Figueira, J an Ernerudh, Magnus Vrethem, Nazdar Ghafouri, Bijar Ghafouri and Malin Nording

The self-archived postprint version of this journal article is available at Linköping University Institutional Repository (DiVA):

http:// urn.kb.se/ resolve?urn=urn:nbn:se:liu:diva- 152634

N.B.: When citing this work, cite the original publication.

Håkansson, I., Gouveia-Figueira, S., Ernerudh, J., Vrethem, M., Ghafouri, N., Ghafouri, B., Nording, M., (2018), Oxylipins in cerebrospinal fluid in clinically isolated syndrome and relapsing remitting multiple sclerosis, Prostaglandins \& other lipid mediators, 138, 41-47.

https:// doi.org/ 10.1016/j.prostaglandins.2018.08.003

Original publication available at:

https:// doi.org/10.1016/j.prostaglandins.2018.08.003

Copyright: Elsevier (12 months)

http://www.elsevier.com/

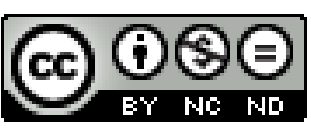


Title:

Oxylipins in cerebrospinal fluid in clinically isolated syndrome and relapsing remitting multiple sclerosis

\section{Authors}

Irene Håkansson ${ }^{1}$, Sandra Gouveia-Figueira ${ }^{2}$, Jan Ernerudh ${ }^{3}$, Magnus Vrethem ${ }^{1}$, Nazdar Ghafouri ${ }^{4}$, Bijar Ghafouri ${ }^{4, *}$, Malin Nording ${ }^{2,5,{ }^{\star}}$

${ }^{1}$ Department of Neurology and Department of Clinical and Experimental Medicine, Linköping University, Linköping, Sweden

${ }^{2}$ Department of Chemistry, Umeå University, Umeå, Sweden

${ }^{3}$ Department of Clinical Immunology and Transfusion Medicine, and Department of Clinical and Experimental Medicine, Linköping University, Linköping, Sweden

${ }^{4}$ Pain and Rehabilitation Centre and Department of Medical and Health Sciences, Linköping University, Linköping, Sweden

${ }^{5}$ Department of Entomology and Nematology, University of California, Davis, USA

* These authors contributed equally to this work.

\section{Corresponding author:}

Irene Håkansson

Department of Neurology

University Hospital in Linköping

58185 Linköping

Sweden

Phone: +46-10-1030000

Fax: +46101034438

E-mail: irene.hakansson@regionostergotland.se 


\section{Highlights:}

- Oxylipin levels in CSF from 41 patients with CIS or RRMS and 22 controls are given.

- CSF levels of 9-HODE and 13-HODE were significantly increased in patients.

- Oxylipin levels were not associated with disease activity during follow-up. 


\section{ABSTRACT}

Although oxylipins are involved in inflammation, data on these lipid mediators in multiple sclerosis are sparse. In this study, a panel of oxylipins were analysed with liquid chromatography tandem mass spectrometry in cerebrospinal fluid (CSF) from 41 treatment naïve patients with clinically isolated syndrome (CIS) or relapsing remitting MS (RRMS) and 22 healthy controls. CSF levels of 9hydroxyoctadecadienoic acid (9-HODE) and 13-hydroxyoctadecadienoic acid (13-HODE) were significantly higher in patients than in healthy controls (9-HODE median $380 \mathrm{nM}$ (interquartile range 330-450 nM) in patients and $290 \mathrm{nM}$ (interquartile range 250-340 nM) in controls, 13-HODE median $930 \mathrm{nM}$ (interquartile range $810-1080 \mathrm{nM}$ ) in patients and $690 \mathrm{nM}$ (interquartile range $570-760 \mathrm{nM}$ ) in controls, $p<0.001$ in Mann-Whitney U-tests). 9-HODE and 13-HODE performed well for separation of patients and healthy controls (AUC 0.85 and 0.88 , respectively, in ROC curve analysis). However, baseline CSF levels of the oxylipins did not differ between patients with signs of disease activity during one, two and four years of follow-up and patients without. In conclusion, this study indicates that 9-HODE and 13-HODE levels are increased in CSF from CIS and RRMS patients compared with healthy controls, but does not support 9-HODE or 13-HODE as prognostic biomarkers of disease activity in patients during follow-up.

Keywords: oxylipins; hydroxyoctadecadienoic acid; mass spectrometry; multiple sclerosis; clinically isolated syndrome 


\section{INTRODUCTION}

Relapsing remitting multiple sclerosis (RRMS) is a chronic inflammatory and neurodegenerative disease of the central nervous system (CNS). Pathology, clinical presentation and disease progression in RRMS is highly variable. Clinically isolated syndrome (CIS) denotes a first episode of neurological symptoms consistent with demyelinating inflammation in the CNS, but where the criteria for multiple sclerosis (MS) diagnosis, which require dissemination in time and space, are not fulfilled. Many patients with CIS later convert to MS, but some do not.

Oxylipins is an umbrella term for fatty acid metabolites involved in maintaining homeostasis. Prostaglandins belong to the eicosanoids, a sub-category of oxylipins derived from arachidonic acid (AA). Other oxylipins are derived from linoleic acid or various other polyunsaturated fatty acids by non-enzymatic oxidation, or cyclooxygenase, lipoxygenase and cytochrome P450 catalyzed reactions. The vast network of bioactive lipid mediators covered by the oxylipin metabolome includes molecules with both pro- and anti-inflammatory properties and several of them have been extensively studied in relation to disease states with an inflammatory component ${ }^{1}$. Mapping this network in a physiological context is challenging, but a better understanding of how altered levels associate with disease may facilitate novel preventive and therapeutic strategies and reveal potential disease biomarkers.

The structural similarities and low abundance of most of the constituents in the oxylipin metabolome have previously been obstacles for quantification in complex human body fluids and tissues. However, recent technological and laboratory advances in lipidomics now enable accurate quantification of these compounds with liquid chromatography combined with tandem mass spectrometry (LC-MS/MS) methods ${ }^{2,3}$. The physiological roles, including pro-inflammatory and antiinflammatory functions, of all the constituents in the oxylipin metabolome have not yet been fully elucidated. Regarding oxylipins in MS patients, there are reports of increased levels of 13hydroxyoctadecadienoic acid (HODE) in plasma ${ }^{4}$ and increased levels of prostaglandin E2 (PGE 2$)$ and 15-hydroxyeicosatetraenoic acid (HETE) in cerebrospinal fluid (CSF) from MS patients compared to controls ${ }^{5}$. Increased levels of PGE 2 and 15-HETE in CSF were also reported in active MS in a 
study comparing more active MS patients to less active MS patients ${ }^{6}$, whereas CSF levels of 4-, 7-, 14- and 17-hydroxydocosahexaenoic acid (HDHA), 5- and 12-HETE, $\mathrm{PGD}_{2}, \mathrm{PGF}_{2}$, lipoxin A4 and B4 ( $\left.\mathrm{LXA}_{4}, \mathrm{LXB} 4\right)$, leukotriene B4 (LTB 4$)$, thromboxane B2 (TXB 2$), \mathrm{AA}$, docosahexaenoic acid (DHA) and eicosapentaenoic acid (EPA) did not differ significantly between these groups ${ }^{6}$.

The aim of this study was to compare levels of a panel of constituents in the CSF oxylipin metabolome in treatment free patients with CIS or RRMS and healthy controls. Furthermore, we explored whether levels of these metabolites correlated to clinical parameters, magnetic resonance imaging (MRI) parameters and a panel of protein markers of neuroinflammation and neurodegeneration. Finally, to assess prognostic biomarker potential, we investigated whether baseline metabolite levels differed between patients that showed signs of disease activity during follow-up and patients that did not. 
MATERIAL AND METHODS

\section{Patients and controls}

41 patients with CIS or RRMS were consecutively enrolled in a prospective longitudinal cohort study of CIS and newly diagnosed MS at the Department of Neurology, University Hospital of Linköping, Sweden. All patients fulfilled the revised McDonald criteria from $2010^{7}$ for CIS or MS. Patients underwent clinical neurological examination including expanded disability status scale (EDSS), peripheral blood and CSF sampling and MRI at baseline and at one, two and four years of follow-up. Only CSF from baseline, when all patients were untreated, was analysed in this study. Patient characteristics are presented in Table 1. For CSF, 22 age- and sex-matched healthy controls were recruited from healthy blood donors. Patients were included in the study from 2009 to 2012 and healthy controls from 2011 to 2014. Healthy controls were free from past and current neurological and autoimmune disease and their clinical neurological examinations were normal as were routine findings in CSF (Table 1). No medication, except oral contraceptives, was allowed in healthy controls.

Table 1 Patient and healthy control characteristics

\begin{tabular}{|c|c|c|c|}
\hline Clinical and laboratory data & $\begin{array}{l}\text { Patients } \\
n=41\end{array}$ & $\begin{array}{l}\text { Healthy controls } \\
n=22\end{array}$ & $\mathrm{p}$ \\
\hline Women/men (\% women) & $32 / 9(78 \%)$ & $17 / 5(77 \%)$ & 0.9 \\
\hline $\mathrm{Age}^{\mathrm{a}}$ (years) & $31(24-36)$ & $32(26-41)$ & 0.3 \\
\hline Diagnosis (CIS/RRMS) & $19 / 22$ & NA & \\
\hline Relapse within last 2 months (yes/no) & $23 / 18$ & NA & \\
\hline Mean disease duration ${ }^{\mathbf{b}}$ (months) & 11.8 & NA & \\
\hline Median disease duration ${ }^{\mathfrak{b}}$ (months) & 3.5 & NA & \\
\hline Disease duration ${ }^{b}$ (number of subjects) & & NA & \\
\hline 0-1 months & 10 & & \\
\hline 1-3 months & 10 & & \\
\hline 3-12 months & 13 & & \\
\hline 13-36 months & 5 & & \\
\hline $37-120$ months & 3 & & \\
\hline Median EDSS & 2.0 & NA & \\
\hline \multicolumn{4}{|l|}{ EDSS (number of subjects) } \\
\hline 0 & 6 & 22 & \\
\hline 1.0 & 12 & & \\
\hline 1.5 & 2 & & \\
\hline $2.0-2.5$ & 15 & & \\
\hline $3.0-4.5$ & 6 & & \\
\hline Chronic pain (number of subjects) & $2^{c}$ & 0 & \\
\hline CSF mononuclear cell count ${ }^{\mathrm{a}}$ & $4.6 \times 10^{6} / \mathrm{L}(1.8-9.2)$ & $2.1 \times 10^{6} / \mathrm{L}(0.9-2.7)$ & 0.001 \\
\hline Albumin ratio ${ }^{a}$ & $4.8(3.4-6.0)$ & $4.7(3.6-5.3)$ & 0.5 \\
\hline $\lg$ index ${ }^{a}$ & $0.7(0.5-1.1)$ & $0.5(0.5-0.5)$ & $<0.001$ \\
\hline Oligoclonal CSF IgG bands (pos/neg) & $33 / 8$ & $0 / 22$ & $<0.001$ \\
\hline Subjects on DMT & 0 & NA & \\
\hline
\end{tabular}


$P$-values from Chi-square test for sex distribution and oligoclonal bands and from Mann-Whitney $U$ test for independent samples for age and CSF data.

${ }^{a}$ Median and within brackets interquartile range.

${ }^{b}$ Disease duration refers to time from first symptom suggestive of demyelinating disease.

${ }^{c}$ One patient had osteoarthritis since several years, with low grade chronic pain in hands, feet and one hip. Another patient had chronic tension type headache and chronic neck and shoulder pain. NA, not applicable

$D M T$, disease-modifying treatment for CIS and RRMS

\section{Ethics Statement}

The study was approved by The Regional Ethics Committee in Linköping and written informed consent was obtained from all participants.

\section{Cerebrospinal fluid sample collection}

All CSF sampling was carried out by the same neurologist $(\mathrm{IH})$ and all lumbar punctures were performed 8-12 a.m. One aliquot of the CSF sample was used for cell counting, CSF/serum albumin ratio, IgG index, IgG synthesis index and isoelectric focusing, all according to clinical routines performed at the Department of Clinical Chemistry. Within one hour, the remaining CSF was centrifuged (300 $\mathrm{xg}$ for $10 \mathrm{~min}$.) and the supernatant was aliquoted and immediately frozen and stored at $-70^{\circ} \mathrm{C}$.

\section{MRI protocol and analyses of protein markers of neuroinflammation and neurodegeneration}

The MRI protocol, as well as the multiplex bead array and the enzyme-linked immunosorbent assays used to determine levels of the neuroinflammatory and neurodegenerative markers, have been described in detail previously8. Mean time from lumbar puncture to MRI was 26 days (range -4 to 64 days).

\section{Measurements of oxylipins in CSF}

Oxylipin levels were determined with liquid chromatography coupled to tandem mass spectrometry in accordance with previously published protocols ${ }^{3}$. The researchers performing the laboratory work were blinded to clinical information. Detailed protocol information is provided in supplementary materials.

\section{Statistics}


Statistical analyses were performed using SPSS for Windows, version 23. Non-parametric tests were mostly used due to the non-Gaussian distribution of some parameters. Values below the detection limit were assigned half the value of the detection limit and included in statistical tests. The MannWhitney U-test was used to compare two groups. Non-parametric bivariate correlation analysis (Spearman) was used when investigating association between lipids and clinical parameters (including EDSS), MRI parameters and protein markers of neuroinflammation and neurodegeneration. Receiver operating characteristic $(\mathrm{ROC})$ curves were derived from logistic regression to investigate the discriminatory power of oxylipins between patients and controls. Because of multiple testing, a stringent $p$-value of $\leq 0.01$ was considered to be significant in the Mann-Whitney U-tests and only correlations with Spearman's rho $\geq 0.5$ were considered to be significant. ANCOVA analysis was used to examine whether significant differences between HODE levels remained when storage time was controlled for. In this ANCOVA, one patient, a strong outlier, was omitted in order to obtain a more parametric distribution of the data, and a significance level of 0.05 was used. All p-values were based on two-tailed statistical tests.

To investigate the multivariate correlations between the oxylipins and group membership, orthogonal partial least squares discriminant analysis (OPLS-DA) was applied using SIMCA-P+ v.14.0 (UMETRICS, Umeå, Sweden) ${ }^{9}$. Principal component analysis (PCA) was used prior to the OPLS-DA analysis. PCA is an unsupervised analysis that can be used to extract and display systematic variation in the data matrix. A cross validation (CV) technique was used to identify nontrivial components. In the OPLS-DA, variables (regressors) were considered important if they had regression coefficients with a jack-knifed 95\% confidence interval not including 0 and the variable of importance (VIP) value was greater than 1. $R^{2}$ describes the goodness of fit - the fraction of sum of squares of all the variables explained by a principal component. $\mathrm{Q}^{2}$ describes the goodness of prediction - the fraction of the total variation of the variables that can be predicted by a principal component using cross validation methods. $\mathrm{R}^{2}$ should not be considerably higher than $\mathrm{Q}^{2}$. A difference greater than 0.2-0.3 implies overfitting, meaning that the robustness of the model is poor ${ }^{9}$. To validate the model, obtained cross validated analysis of variance (CV-ANOVA) was used. The multivariate regressions were considered of significant importance if the CV-ANOVA had $p<0.05$. The 
procedure to compute multivariate correlation models is in accordance with Wheelock and Wheelock ${ }^{10}$. 
RESULTS

\section{Oxylipins in patients and healthy controls}

9-HODE and 13-HODE levels were significantly higher in patients than in healthy controls, both p $<0.001$ (Table 2 and Figure 1). Excluding the two patients with chronic pain did not change this finding (both $p$ still $<0.001$ ). Samples from patients had longer storage times than samples from healthy controls, but ANCOVA analysis showed that there was a significant difference between HODE levels in patients and healthy controls also when storage time was controlled for. 9-HODE and 13HODE levels correlated significantly in patients (Spearman's rho 0.55, $p<0.001$ ), whereas in healthy controls Spearman's rho was $0.47, p=0.03$. All analytes were detected in CSF samples from all subjects, except PGE 2 and 15-HETE, which were not detected in any subject.

Table 2 Oylipins in CSF from patients with CIS or RRMS and from healthy controls

\begin{tabular}{llll}
\hline & Patients $(\mathrm{n}=41)$ & Healthy controls $(\mathrm{n}=22)$ & $\mathrm{p}$ \\
\hline 11,12-DHET & $24(20-27)$ & $22(16-33)$ & 0.55 \\
14,15-DHET & $52(43-63)$ & $52(43-78)$ & 0.65 \\
15-HETE & ND & ND & \\
9-HODE & $380(330-450)$ & $290(250-340)$ & $<0.001$ \\
13-HODE & $930(810-1080)$ & $690(570-760)$ & $<0.001$ \\
9,10-DiHOME & $55(49-71)$ & $57(49-64)$ & 0.59 \\
12,13-DiHOME & $91(77-110)$ & $84(78-98)$ & 0.32 \\
9,10,13-TriHOME & $190(160-210)$ & $180(170-200)$ & 0.98 \\
9,12,13-TriHOME & $580(530-660)$ & $580(530-700)$ & 0.72 \\
PGE 2 & ND & ND & \\
PGF $2 a$ & $165(120-240)$ & $225(140-350)$ & 0.07 \\
TXB 2 & $63(56-74)$ & $55(47-71)$ & 0.12 \\
\hline
\end{tabular}

Data are expressed as median and within brackets interquartile range and concentrations of oxylipins are given in nM. P-values from Mann-Whitney U-test. ND: Not detectable. DHET: Dihydroxyeicosatrienoic acid; HETE: Hydroxyeicosatetraenoic acid; HODE: Hydroxyoctadecadienoic acid; DiHOME: Dihydroxyoctadecenoic acid; TriHOME: Trihydroxyoctadecenoic acid; $P G E_{2}$ Prostaglandin $E_{2} ; P G F_{2 a}$ : Prostaglandin $F_{2 \alpha} ; T X B_{2}$ : Thromboxane $B_{2}$ 

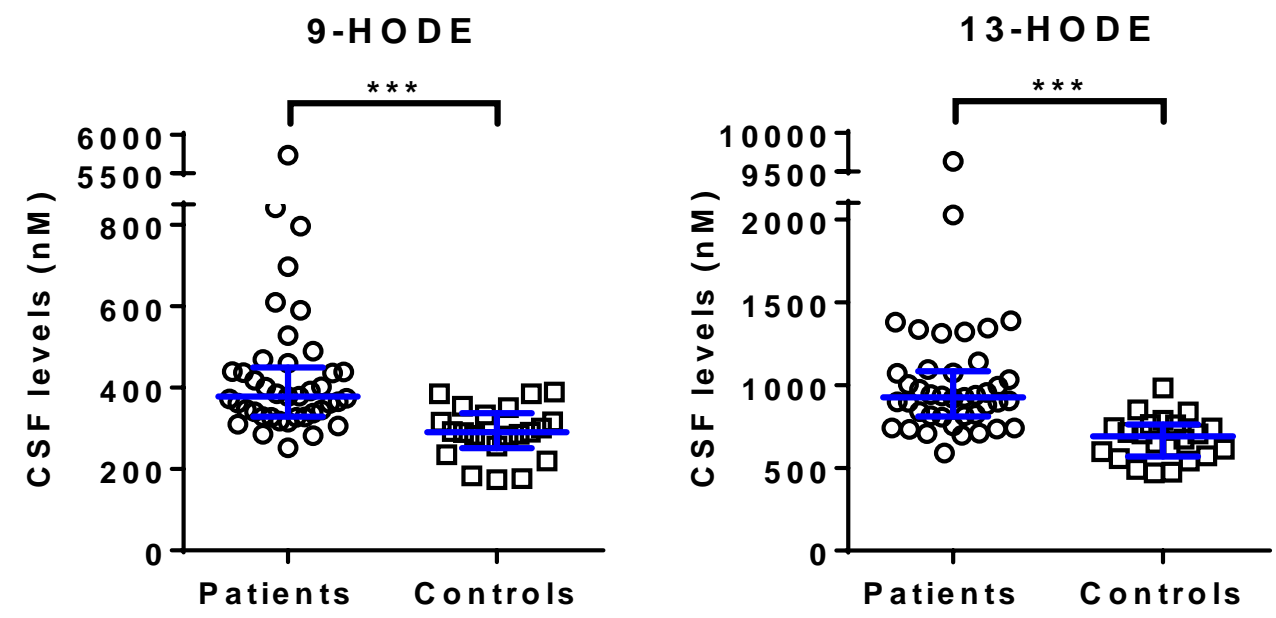

Figure 1 Levels of 9-HODE and 13-HODE in CSF from patients with CIS or RRMS and from healthy controls. Lines show median values and interquartile range.

*** $p<0.001$ in Mann-Whitney U-test.

\section{Oxylipins for separation of patients and healthy controls}

ROC curve analysis was performed on 9-HODE and 13-HODE to investigate their ability to discriminate between patients and healthy controls. ROC curves and area under curve (AUC) values are presented in Figure 2. For comparison, the AUC value for neurofilament light chain in CSF for separation of the same patients and healthy controls was 0.85 , as previously reported 8 .
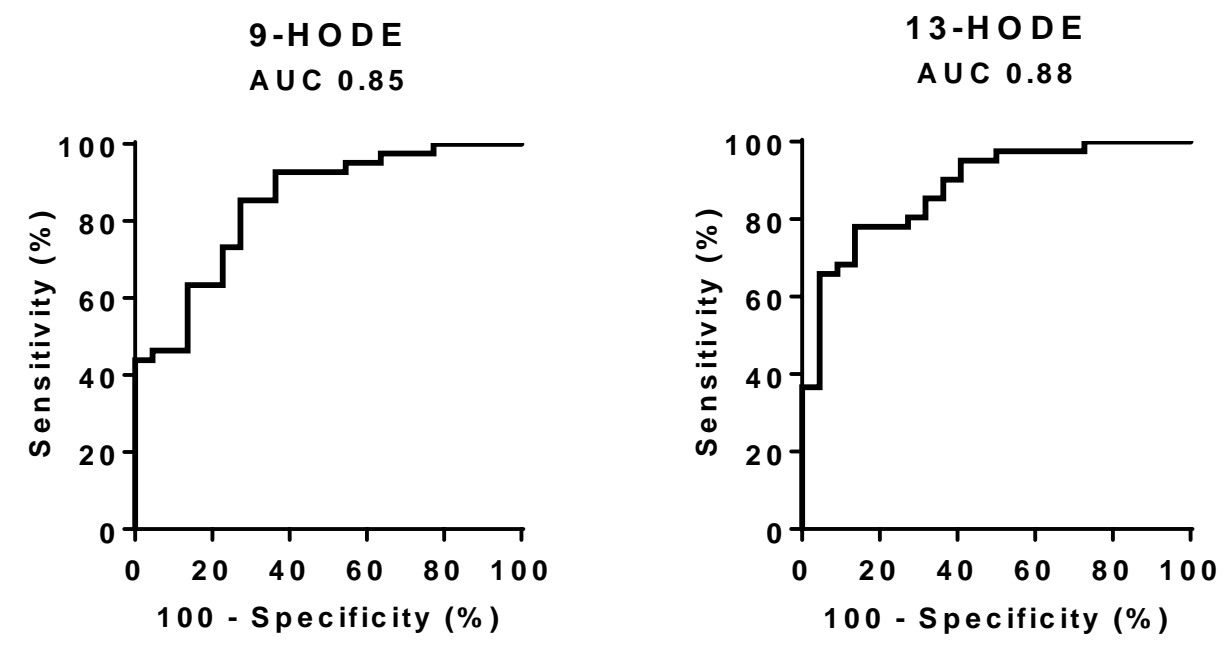
Figure 2 Receiver operating characteristics (ROC) curves for 9-HODE and 13-HODE in CSF at baseline for separation of patients with CIS or RRMS $(n=41)$ and healthy controls $(n=22)$. Area under curve (AUC) values are given in the figure.

\section{Oxylipins in relation to clinical, MRI and CSF parameters at baseline}

11,12-DHET was higher in men than in women $(p=0.003)$ in the patient group (Figure $3 A)$. Apart from that, lipid levels did not differ significantly between women and men in patients or healthy controls. Age did not correlate significantly with lipid levels in patients or healthy controls, except for PGF $2 a$ in healthy controls (Spearman's rho 0.54, $\mathrm{p}=0.01$ ).

Patients in relapse (time from onset of relapse within two months before CSF collection) ( $n=23)$ had significantly higher levels of 11,12-DHET $(\mathrm{p}=0.007)$ (Figure 3B) and a trend towards higher levels of $14,15-\mathrm{DHET}(\mathrm{p}=0.019)$ than non-relapse patients $(n=18)$. The percentage of persons in relapse at the time of sample collection was the same, $56 \%$, among male and female patients. Relapse status did not affect levels of the other lipids. None of the analyzed lipids differed significantly between CIS patients $(n=19)$ and RRMS patients $(n=22)($ all $p>0.1)$. Baseline EDSS score, disease duration, number of T2 hyperintense lesions in brain MRI, mononuclear cell count in CSF and IgG index did not correlate significantly with any of the analyzed lipids (all Spearman's rho <0.5).

A

11,12 -D HET

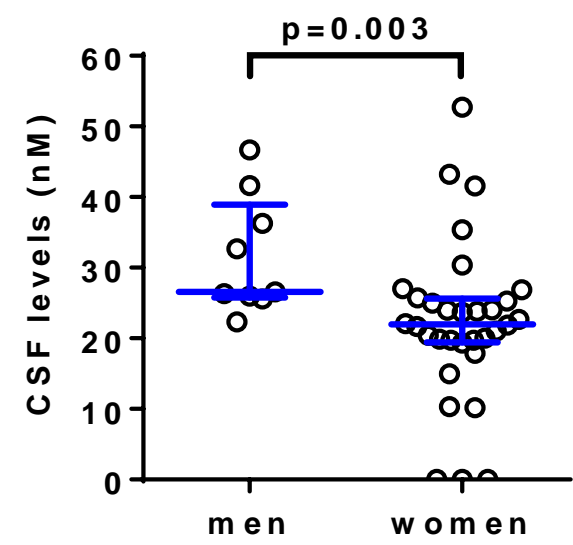

B

\section{1,12-D HET}

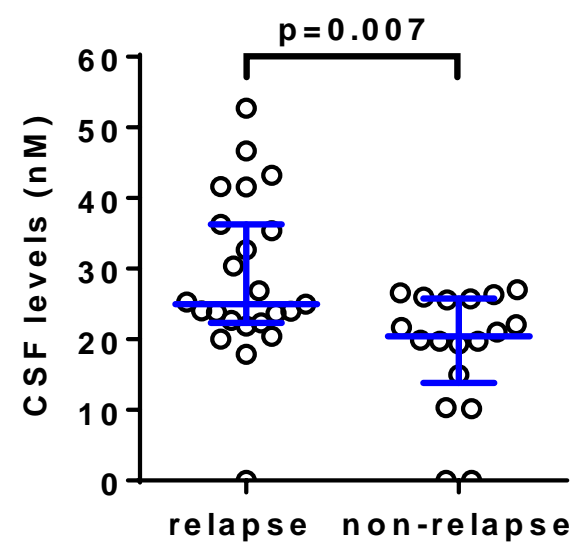

Figure 3 Levels of 11,12-DHET in CSF from male and female patients (A) and from patients in relapse and not in relapse (B) at the time of sample collection. The percentage of persons in relapse at the time of sample collection was the same (56\%) among male and female patients. 
Lines show median values and interquartile range.

P-values from Mann-Whitney U-test for independent samples.

\section{Oxylipins in relation to protein neuroinflammatory and neurodegenerative markers at baseline}

Baseline CSF levels of the chemokines CXCL1, CXCL8, CXCL10, CXCL13 and CCL22 from the patients and healthy controls in this study have previously been reported, as well as baseline CSF levels of neurofilament light chain (NFL), neurofilament heavy chain (NFH), matrix metalloproteinase 9 (MMP-9), glial fibrillary acidic protein (GFAP), chitinase 3 like 1 (CHI3L1) and osteopontin (OPN) ${ }^{8}$.

In correlation analyses based on data from all subjects, i.e. pooled data from patients and healthy controls, 13-HODE correlated significantly with CXCL8 and CCL22 with our strict significance level of Spearman's rho $\geq 0.50$ and $p \leq 0.001$ (Table 3). Several correlation coefficients between 0.40 and 0.49 were noted, for 9-HODE with CXCL10 and CXCL13, CCL22 and MMP-9 and for 13-HODE with CXCL10 and NFL (Table 3). There were no significant correlations, and also no correlation coefficients between 0.40 and 0.49 , between baseline CSF levels of $\mathrm{PGF}_{2 \alpha}, \mathrm{TXB}_{2}, 11,12$-DHET, 14,15-DHET, 9-HODE, 9,10-DiHOME,12,13-DiHOME, 9,10,13-TriHOME or 9,12,13-TriHOME and the proteins. When data from patients and healthy controls were analyzed separately, no significant correlations where present in either the patient group or in the healthy control group.

Table 3 Correlations between CSF levels of protein neuroinflammatory and neurodegenerative markers and 9-HODE and 13-HODE, respectively, in all subjects $(n=63)$.

\begin{tabular}{lll}
\hline & $9-H O D E$ & 13-HODE \\
\hline CXCL1 & 0.24 & 0.39 \\
CXCL8 & 0.39 & $\mathbf{0 . 5 4}$ \\
CXCL10 & $\mathbf{0 . 4 0}$ & $\mathbf{0 . 4 7}$ \\
CXCL13 & $\mathbf{0 . 4 4}$ & 0.34 \\
CCL22 & $\mathbf{0 . 4 0}$ & $\mathbf{0 . 5 0}$ \\
NFL & 0.33 & $\mathbf{0 . 4 4}$ \\
NFH & 0.12 & 0.17 \\
GFAP & 0.12 & 0.12 \\
CHI3L1 & 0.22 & 0.23 \\
MMP-9 & $\mathbf{0 . 4 9}$ & 0.37 \\
OPN & 0.19 & 0.09 \\
\hline
\end{tabular}




\begin{abstract}
Magnitude of correlation coefficients from bivariate
non-parametric correlation analyses (Spearman) are

shown, with correlation coefficients $\geq 0.4$ in bold.

*: Spearman's rho $\geq 0.50$ and $p \leq 0.001$
\end{abstract}

\title{
Multivariate data analysis
}

PCA was performed in order to identify multivariate outliers. The PCA score plots in combination with Hotelling's T2 (identifies strong outliers) and distance to model in X-space (identifies moderate outliers) showed one strong outlier and that patient was excluded from further analyses. To identify the substance important for group separation of patients and healthy controls an OPLS-DA regression model was generated (figure 4). A total of 10 substances with VIP value $>1$ were considered important for group separation and they are presented in figure 5. These oxylipins and proteins together explained $44 \%\left(\mathrm{R}^{2}\right)$ of the variation and with a prediction of $30 \%\left(\mathrm{Q}^{2}\right)$. The CV-ANOVA revealed that the model was highly significant $(p=0.002)$. Among the strongest discriminating substances were the oxylipins 9-HODE and 13-HODE, with VIP values of approximately 1.5, which were more abundant in patients than in healthy controls. This difference in concentration was furthermore found to be significant (using univariate statistical analysis), independently of other factors, indicating that these factors constitute some of the key differences seen between patients and controls.

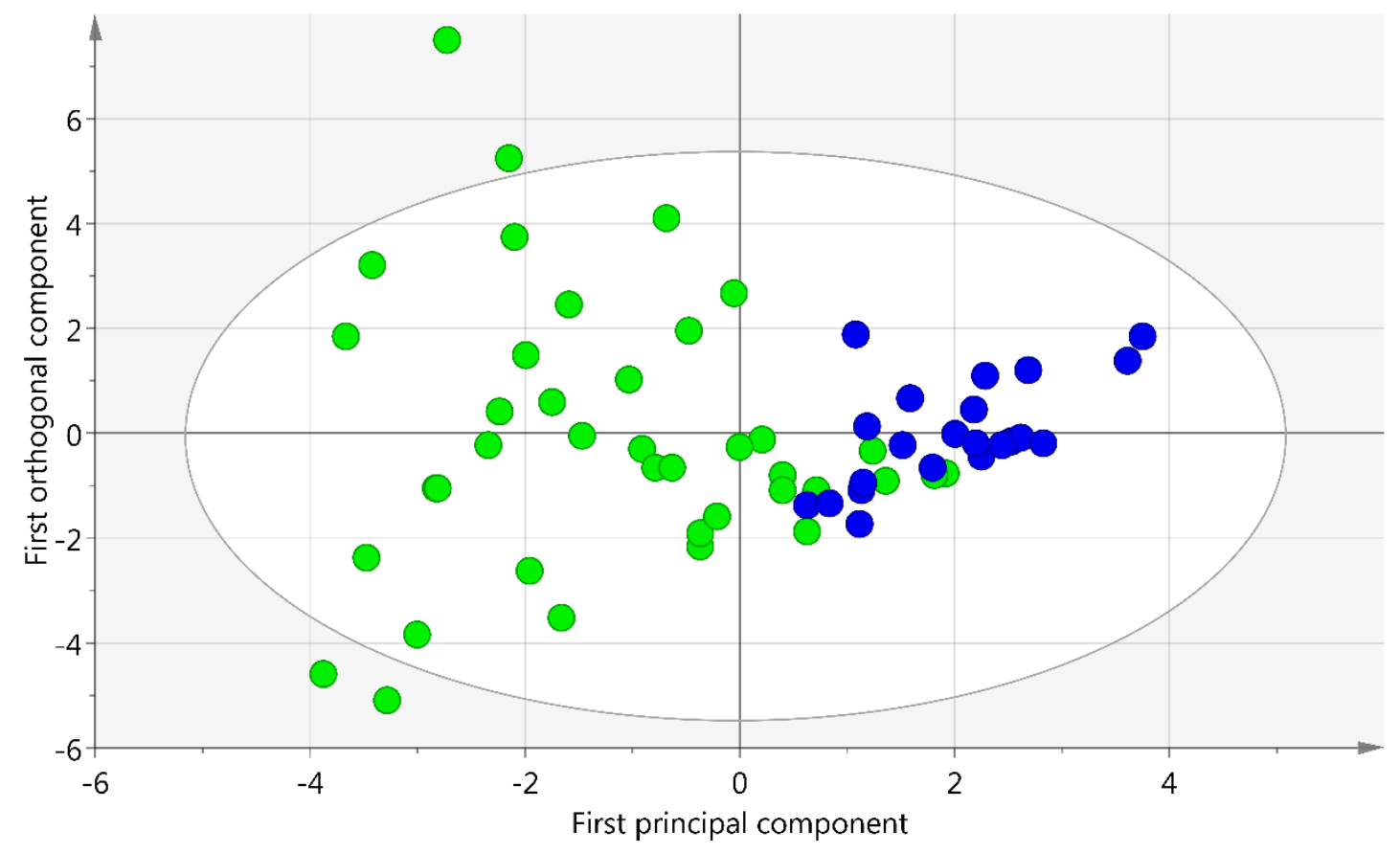


Figure 4. An OPLS-DA (first principal component t1 vs. the first orthogonal component to1) model showing the discriminant separation between the patients (green filled circles) and the controls (blue filled circles). The longitudinal dimension (X-axis) shows the interclass discrimination and the latitudinal dimension (Y-axis) shows the intraclass discrimination between patients and controls.

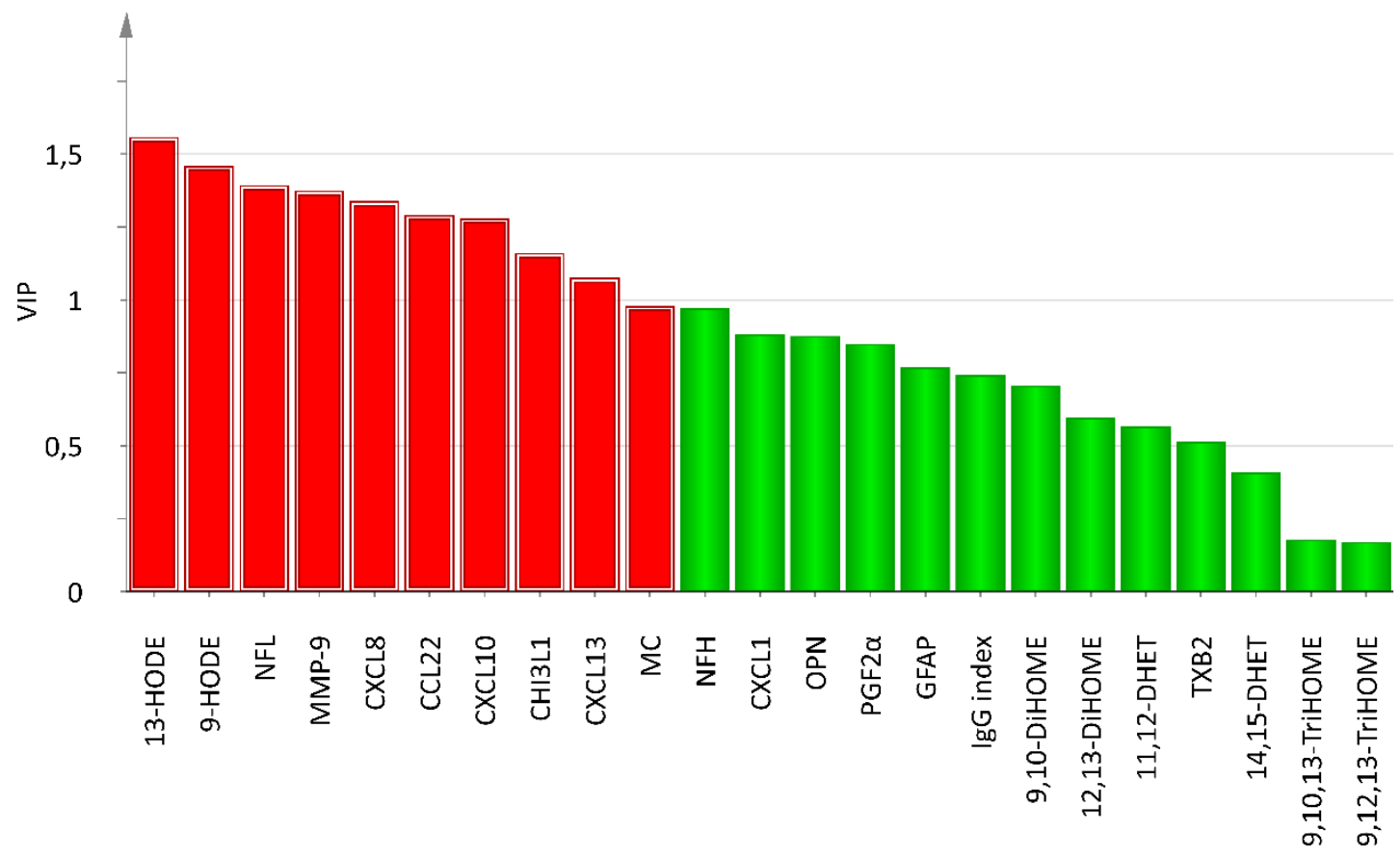

Figure 5. Variable importance of projection (VIP) values for oxylipins and proteins. VIP values $>1.0$ (red bars) were considered significant. 13-HODE and 9-HODE were the most important substances for group separation (patients vs. controls). MC: mononuclear cell count in CSF

\section{Baseline oxylipin levels in relation to disease activity during follow-up}

All 41 patients were evaluated at one year, 39 patients at two years (one patient did not undergo an MRI due to pregnancy and one patient had left the study) and 39 patients at four years (two patients had left the study). Evidence of disease activity during follow-up was defined as relapses, brain MRI activity (new or enlarging T2 lesions or Gadolinium-enhancing lesions) or sustained disability worsening (EDSS progression). There were no significant differences in CSF levels of the lipids at baseline between patients with evidence of disease activity during one $(n=21)$, two $(n=27)$ and four 
$(n=32)$ years of follow-up, and patients with no evidence of disease activity (NEDA-3) during follow-up ( $n=20$ at one year, $n=12$ at two years and $n=7$ at four years). 


\section{DISCUSSION}

We report that CSF levels of 9-HODE and 13-HODE were significantly higher in patients with CIS and RRMS than in healthy controls. Patients in relapse had significantly higher levels of 11,12-DHET than patients not in relapse at the time of sample collection and this lipid was also significantly higher in men compared to women in the patient group. 9-HODE and 13-HODE performed well at separating patients and healthy controls and also correlated with several protein markers of neuroinflammation and neurodegeneration when data from all subjects were analyzed. However, in the patient group, there were no significant correlations between oxylipins and protein markers at baseline and there were also no significant differences in CSF oxylipin levels at baseline between patients with and patients without evidence of disease activity during follow-up.

An essential feature of the innate immune system is maintaining cellular homeostasis by identifying and removing senescent and apoptotic cells and modified lipids on the surface of cell membranes and lipoprotein particles ${ }^{11}$. As cellular membranes undergo lipid peroxidation, previously hydrophobic portions of fatty acids will move from the interior of the lipid bilayer to the aqueous exterior enabling physical contact between pattern recognition receptors and molecular pattern ligands and lipid peroxidation has been shown to induce disturbance of fine structures, alteration of integrity, fluidity, and permeability, and functional loss of biomembranes ${ }^{11}$. The formation of lipid peroxidation products must be strictly controlled and programmed in order to exert physiologically important functions, such as regulation of gene expression and mediation of cellular signaling ${ }^{12}$. Accumulation of many of the lipid peroxidation products are known to be toxic for cells and the CNS is particularly vulnerable to uncontrolled lipid peroxidation ${ }^{13}$. Increased lipid peroxidation in patients with MS has previously been suggested and challenged $14,15,16,17,18,19,20$. HODEs are considered as nearly ideal markers for lipid peroxidation ${ }^{21}$. To our knowledge, there are no previous reports on 9-HODE or 13-HODE levels in CSF in CIS or RRMS patients. It has recently been reported that higher levels of 13-HODE in serum were part of a metabolomic signature associated with relapses and increase in EDSS in MS4. However, CSF was not analyzed in that study. It should be noted that alterations in HODE levels are not restricted to MS. As for reports on HODE levels in CSF in other neurological diseases, 9-HODE levels in children with brain tumors ${ }^{22}$ and hydrocephalus ${ }^{23}$ did not differ from controls. Outside the 
central nervous system, HODEs have been implicated in various contexts, e.g. in the inflammatory process in atherosclerosis ${ }^{24}$, in the metabolic syndrome and some cancers ${ }^{25}$, in perceived pain in individuals with localized musculoskeletal pain ${ }^{26}$ and Achilles tendinopathy ${ }^{27}$, and as oxidative stress biomarkers in acute exercise ${ }^{28}$.

The increased CSF levels of 9-HODE and 13-HODE in CIS and RRMS patients compared to controls in our study may reflect inflammation in the CNS in the patient group. The HODEs have pleiotropic effects in humans. Actions that may be relevant in the context of MS inflammation are that the HODEs, as well as 15-HETE, activate the peroxisome proliferator-activated receptor gamma (PPARY) receptor, thereby influencing the maturation, differentiation and activation of monocytes, macrophages and dendritic cells ${ }^{29}$. PPARy also interacts with transcription factors involved in proinflammatory signaling pathways, such as NF-B and STAT1, thereby repressing pro-inflammatory responses in e.g. activated T cells ${ }^{30}$. These are potentially anti-inflammatory effects. On the other hand, HODEs are chemotactic agents for neutrophils and natural killer cells in vitro ${ }^{31,32}$, which could suggest pro-inflammatory effects of HODEs in MS. The effects of lipid peroxidation products might be concentration dependent since although many of them exert cytotoxicity, sublethal concentrations of such products, including HODEs, induce cellular adaptive responses and enhance tolerance against subsequent oxidative stress suggesting both deleterious and beneficial effects of lipid peroxidation in vivo $^{12}$.

$\mathrm{PGE}_{2}$ and 15-HETE in CSF have been reported to be higher in highly active MS compared to less active $\mathrm{MS}^{6}$ and to be higher in MS patients compared to controls ${ }^{5}$, whereas levels of $\mathrm{TXB}_{2}$ in CSF did not differ between highly active and less active disease ${ }^{6}$. TXB 2 was not associated with disease activity in our study either. Median PGE 2 was $430 \mathrm{ng} / \mathrm{L}$ in patients and $216 \mathrm{ng} / \mathrm{L}$ in controls and median 15-HETE was $646 \mathrm{ng} / \mathrm{L}$ in patients and $565 \mathrm{ng} / \mathrm{L}$ in controls in the study reporting higher levels of these analytes in MS patients than in controls $5 . \mathrm{PGE}_{2}$ and 15-HETE were not detectable in our patients and controls.

The overall lack of association between the baseline oxylipin levels and disease activity parameters in patients at baseline and during follow-up in our study suggests that, although levels of two of them 
differ between patients and healthy controls, these oxylipins may not be central in the specific pathophysiology of MS, at least not linked to degree of disease activity. Rather, increased HODE levels in patients may be an unspecific sign of neuroinflammation. On the other hand, oxylipins are produced on demand, act locally, are metabolized differently and are more sensitive to pre-analytical influences than protein markers and therefore levels in CSF may not reflect disease activity as well as e.g. NFL. These remarks are only speculative, and it is to be recognized that this is an observational study that does not allow conclusions about causality or pathophysiology. However, we do claim that none of the lipids in our panel shows potential as a prognostic biomarker in MS, based on that we do not see an association with NEDA-3 during follow-up and the lack of significant correlations with accepted prognostic biomarkers like NFL in the patient group. While both NFL and the hydroxyoctadecadienoic acids show significant differences between patients and healthy controls, NFL also conveys prognostic information in the patient group ${ }^{8}$, whereas the lipids do not. In future studies, it would be of interest to measure oxylipins in MS patients repeatedly during follow-up and to evaluate possible associations with pain, spasticity and cognitive impairment in addition to NEDA-3.

Strengths of our study are the well characterized and treatment free patient group and the age- and sex-matched healthy control group, as well as availability of data on protein neuroinflammatory and neurodegenerative markers and follow-up data on disease activity. Limitations are the relatively small number of patients and the large number of comparisons made in some of the statistical analyses. We used strict significance levels to lower the risk of false positive findings, but further studies are needed to verify our findings. Also, HODE levels vary with dietary intake of linoleic acid ${ }^{33}$ and we cannot exclude diet as a potential confounding factor.

In conclusion, this study indicates that levels of 9-HODE and 13-HODE in CSF are increased in patients with CIS and RRMS. We found no association between baseline levels of the oxylipins analyzed and disease activity during follow-up. 


\section{REFERENCES}

1. Dennis EA and Norris PC. Eicosanoid storm in infection and inflammation. Nat Rev Immunol. 2015; 15: 511-23.

2. Balgoma D, Checa A, Sar DG, Snowden S and Wheelock CE. Quantitative metabolic profiling of lipid mediators. Mol Nutr Food Res. 2013; 57: 1359-77.

3. Gouveia-Figueira S and Nording ML. Validation of a tandem mass spectrometry method using combined extraction of 37 oxylipins and 14 endocannabinoidrelated compounds including prostamides from biological matrices. Prostaglandins Other Lipid Mediat. 2015; 121: 110-21.

4. Villoslada P, Alonso C, Agirrezabal I, et al. Metabolomic signatures associated with disease severity in multiple sclerosis. Neurol Neuroimmunol Neuroinflamm. 2017; 4: e321.

5. Mattsson N, Yaong M, Rosengren L, et al. Elevated cerebrospinal fluid levels of prostaglandin E2 and 15-(S)-hydroxyeicosatetraenoic acid in multiple sclerosis. J Intern Med. 2009; 265: 459-64.

6. Pruss H, Rosche B, Sullivan AB, et al. Proresolution lipid mediators in multiple sclerosis - differential, disease severity-dependent synthesis - a clinical pilot trial. PLoS One. 2013; 8: e55859.

7. Polman CH, Reingold SC, Banwell B, et al. Diagnostic criteria for multiple sclerosis: 2010 revisions to the McDonald criteria. Ann Neurol. 2011; 69: 292-302.

8. Hakansson I, Tisell A, Cassel P, et al. Neurofilament light chain in cerebrospinal fluid and prediction of disease activity in clinically isolated syndrome and relapsing-remitting multiple sclerosis. Eur J Neurol. 2017; 24: 703-12.

9. $\quad$ Eriksson L, et al. Multi- and Megavariate Data analysis; part I and II. 2 ed.: Umetrics AB, 2006.

10. Wheelock AM and Wheelock CE. Trials and tribulations of 'omics data analysis: assessing quality of SIMCA-based multivariate models using examples from pulmonary medicine. Mol Biosyst. 2013; 9: 2589-96.

11. Greenberg ME, Li XM, Gugiu BG, et al. The lipid whisker model of the structure of oxidized cell membranes. J Biol Chem. 2008; 283: 2385-96.

12. Niki E. Lipid peroxidation: physiological levels and dual biological effects. Free Radic Biol Med. 2009; 47: 469-84.

13. Anthonymuthu TS, Kenny EM and Bayir H. Therapies targeting lipid peroxidation in traumatic brain injury. Brain Res. 2016; 1640: 57-76.

14. Toshniwal PK and Zarling EJ. Evidence for increased lipid peroxidation in multiple sclerosis. Neurochemical research. 1992; 17: 205-7.

15. Hunter MI, Nlemadim BC and Davidson DL. Lipid peroxidation products and antioxidant proteins in plasma and cerebrospinal fluid from multiple sclerosis patients. Neurochemical research. 1985; 10: 1645-52.

16. Koch M, Mostert J, Arutjunyan AV, et al. Plasma lipid peroxidation and progression of disability in multiple sclerosis. Eur J Neurol. 2007; 14: 529-33.

17. Naidoo R and Knapp ML. Studies of lipid peroxidation products in cerebrospinal fluid and serum in multiple sclerosis and other conditions. Clin Chem. 1992; 38: 2449-54.

18. Ljubisavljevic S, Stojanovic I, Pavlovic D, et al. Suppression of the lipid peroxidation process in the CNS reduces neurological expression of experimentally induced autoimmune encephalomyelitis. Folia Neuropathol. 2013; 51: 51-7. 
19.

Pasichna EP, Morozova RP, Donchenko HV, Vinychuk SM and Kopchak OO.

[Lipid peroxidation and antioxidant defence enzyme activity in multiple sclerosis]. $U k r$ Biokhim Zh (1999). 2007; 79: 165-74.

20. Sosa RA, Murphey C, Robinson RR and Forsthuber TG. IFN-gamma ameliorates autoimmune encephalomyelitis by limiting myelin lipid peroxidation. Proc Natl Acad Sci U S A. 2015; 112: E5038-47.

21. Spiteller P and Spiteller G. 9-Hydroxy-10,12-octadecadienoic acid (9-HODE) and 13-hydroxy-9,11-octadecadienoic acid (13-HODE): excellent markers for lipid peroxidation. Chemistry and Physics of Lipids. 1997; 89: 131-9.

22. Cengiz P, Zemlan F, Eickhoff JC, Ellenbogen R and Zimmerman JJ. Increased cerebrospinal fluid cleaved tau protein (C-tau) levels suggest axonal damage in pediatric patients with brain tumors. Childs Nerv Syst. 2015; 31: 1313-9.

23.

Cengiz P, Zemlan F, Ellenbogen R, Hawkins D and Zimmerman JJ.

Cerebrospinal fluid cleaved-tau protein and 9-hydroxyoctadecadienoic acid concentrations in pediatric patients with hydrocephalus. Pediatr Crit Care Med. 2008; 9: 524-9.

24. Rolin J and Maghazachi AA. Implications of chemokines, chemokine receptors, and inflammatory lipids in atherosclerosis. J Leukoc Biol. 2014; 95: 575-85.

25. Vangaveti VN, Jansen H, Kennedy RL and Malabu UH.

Hydroxyoctadecadienoic acids: Oxidised derivatives of linoleic acid and their role in inflammation associated with metabolic syndrome and cancer. Eur J Pharmacol. 2016; 785: 70-6.

26. Hellstrom F, Gouveia-Figueira S, Nording ML, Bjorklund M and Fowler CJ. Association between plasma concentrations of linoleic acid-derived oxylipins and the perceived pain scores in an exploratory study in women with chronic neck pain. BMC Musculoskelet Disord. 2016; 17: 103.

27. Gouveia-Figueira S, Nording ML, Gaida JE, Forsgren S, Alfredson H and Fowler CJ. Serum levels of oxylipins in achilles tendinopathy: an exploratory study. PLoS One. 2015; 10: e0123114.

28. Nieman DC, Shanely RA, Luo B, Meaney MP, Dew DA and Pappan KL. Metabolomics approach to assessing plasma 13- and 9-hydroxy-octadecadienoic acid and linoleic acid metabolite responses to 75-km cycling. Am J Physiol Regul Integr Comp Physiol. 2014; 307: R68-74.

29. Kiss M, Czimmerer Z and Nagy L. The role of lipid-activated nuclear receptors in shaping macrophage and dendritic cell function: From physiology to pathology. $J$ Allergy Clin Immunol. 2013; 132: 264-86.

30. Glass CK and Saijo K. Nuclear receptor transrepression pathways that regulate inflammation in macrophages and T cells. Nat Rev Immunol. 2010; 10: 365-76.

31. Henricks PA, Engels F, van der Vliet H and Nijkamp FP. 9- and 13-hydroxylinoleic acid possess chemotactic activity for bovine and human polymorphonuclear leukocytes. Prostaglandins. 1991; 41: 21-7.

32. $\quad$ Rolin J, Al-Jaderi Z and Maghazachi AA. Oxidized lipids and lysophosphatidylcholine induce the chemotaxis and intracellular calcium influx in natural killer cells. Immunobiology. 2013; 218: 875-83.

33. Ramsden CE, Ringel A, Feldstein AE, et al. Lowering dietary linoleic acid reduces bioactive oxidized linoleic acid metabolites in humans. Prostaglandins Leukot Essent Fatty Acids. 2012; 87: 135-41. 


\section{SUPPLEMENTARY MATERIAL}

\section{Measurements of oxylipins in CSF}

A previously validated solid phase extraction (SPE) protocol was modified to isolate oxylipins from CSF samples ${ }^{3}$. In summary, Waters Oasis HLB cartridges (60 mg of sorbent, $30 \mu \mathrm{m}$ particle size) were first washed with ethyl acetate $(1 \mathrm{~mL})$ and methanol $(2 \times 2 \mathrm{~mL})$, then conditioned with $5 \%$ methanol in water (containing $0.1 \%$ acetic acid) before the CSF sample was loaded. Quantitative volumes from each sample $(\sim 0.3 \mathrm{~mL})$ were spiked with a $10 \mu \mathrm{L}$ internal standard mixture in methanol with the following concentrations, $50 \mathrm{ng} / \mathrm{mL}$ 12(13)-DiHOME- $\mathrm{d}_{4}$ and 12(13)-EPOME- $\mathrm{d}_{4}$, and $25 \mathrm{ng} / \mathrm{mL}$ 9-HODE- $\mathrm{d}_{4}, \mathrm{PGE}_{2}-\mathrm{d}_{4}$ and $\mathrm{TXB}_{2}-\mathrm{d}_{4}$, , as well as $10 \mu \mathrm{L}$ antioxidant solution $(0.2 \mathrm{mg} / \mathrm{mL}$ BHT/EDTA in methanol/water (1:1)). After the samples were applied, the SPE cartridges were washed, dried under high vacuum and eluted with $3 \mathrm{~mL}$ acetonitrile, $2 \mathrm{~mL}$ methanol and $1 \mathrm{~mL}$ ethyl acetate. To trap the analytes, all three fractions were pooled into polypropylene tubes containing $6 \mu \mathrm{L}$ of a glycerol solution (30\% in methanol) and evaporated using a MiniVac system (Farmingdale, NY, U.S.A.), then reconstituted in $100 \mu \mathrm{L}$ of methanol, spiked with $10 \mu \mathrm{L}$ recovery standard ( $50 \mathrm{ng} / \mathrm{mL}$ CUDA), transferred to vials, and analyzed by LC-MS/MS. The Agilent Ultra-Performance LC system (Infinity 1290) coupled to an Agilent 6490 Triple Quadrupole mass spectrometer with an electrospray ionization source (ESI) equipped with the iFunnel Technology (Agilent Technologies, Santa Clara, CA, USA) was used for separation and detection of the analytes. From the LC vials, $10 \mu \mathrm{L}$ was injected on a Waters BEH C18 column (2.1 mm x $150 \mathrm{~mm}, 130 \AA$, $1.7 \mu \mathrm{m}$ particle size). and the mobile phase used for elution of the analytes was composed of (A) $0.1 \%$ acetic acid in deionized water and (B) acetonitrile:isopropanol (90:10) with the following gradient:0.0-3.5 min 10-35\% B, 3.5$5.5 \min 35-40 \%$ B, $5.5-7.0 \min 42 \%$ B, $7.0-9.0 \min 50 \%$ B, $9.0-15.0 \min 65 \%$ B, $15.0-17.0 \min 75 \%$ B, $17.0-18.5 \min 85 \%$ B, $18.5-19.5 \min 95 \%$ B, $19.5-21.0 \min 10 \%$ B, 21.0-25.0 min 10\% B.

The ESI-MS/MS conditions for operating the ESI in negative mode were the following: capillary and nozzle voltage at $4000 \mathrm{~V}$ and $1500 \mathrm{~V}$, drying gas temperature $230^{\circ} \mathrm{C}$ with a gas flow of $15 \mathrm{~L} / \mathrm{min}$, sheath gas temperature $400{ }^{\circ} \mathrm{C}$ and gas flow of $11 \mathrm{~L} / \mathrm{min}$; the nebulizer gas flow was set at $35 \mathrm{psi}$, and iFunnel high and low pressure RF were 150 and $60 \mathrm{~V}$. Dynamic multiple reaction monitoring (MRM) mode was used with fixed time windows (retention time $\pm 2 \mathrm{~min}$ ) to profile one quantitative and one qualitative transition per analyte ${ }^{3}$. The dynamic MRM option was performed for all analytes with 
optimized transitions and collision energies. The MassHunter Workstation software was used for instrument control and for subsequent manual integration of the peaks. Native standards (TXB 9,12,13-TriHOME, 9,10,13-TriHOME, PGF2a, PGE2, 12,13-DiHOME, 9,10-DiHOME, 14,15-DHET, 11,12-DHET, 13-HODE, 9-HODE, and 15-HETE) and internal standards (TXB ${ }_{2}-\mathrm{d}_{4}, \mathrm{PGE}_{2}-\mathrm{d}_{4}, 12,13-$ DiHOME- $\mathrm{d}_{4}, 20-\mathrm{HETE}-\mathrm{d}_{6}, 9-\mathrm{HODE}-\mathrm{d}_{4}, \mathrm{~d}_{8}-5-\mathrm{HETE}$, and 12(13)-EpOME- $\left.\mathrm{d}_{4}\right)$ were purchased from Cayman Chemicals (Ann Arbor, USA). The limit of detection was $2.7 \mathrm{pM}$ for $\mathrm{TXB}_{2}, 0.15 \mathrm{pM}$ for 9,12,13-TriHOME, 3 pM for 9,10,13-TriHOME, 3 pM for PGE 2,3 pM for PGF $2 \alpha, 3.5$ pM for 12,13DiHOME, 0.35 pM for 9,10-DiHOME, 3.25 pM for 14,15-DHET, 16 pM for 11,12-DHET, 18.5 pM for 13-HODE $0.4 \mathrm{pM}$ for 9-HODE and $3.4 \mathrm{pM}$ for 15-HETE. 